Meta

Journal des tradlucteurs

Translators' Journal

\title{
Rouleau, M. (2001) : Initiation à la traduction générale. Du mot au texte, Brossard, Linguatech, $241 \mathrm{p}$.
}

\section{Jean-Claude Gémar}

Volume 48, numéro 4, décembre 2003

URI : https://id.erudit.org/iderudit/008743ar

DOI : https://doi.org/10.7202/008743ar

Aller au sommaire du numéro

\section{Éditeur(s)}

Les Presses de l'Université de Montréal

\section{ISSN}

0026-0452 (imprimé)

1492-1421 (numérique)

Découvrir la revue

Citer ce compte rendu

Gémar, J.-C. (2003). Compte rendu de [Rouleau, M. (2001) : Initiation à la

traduction générale. Du mot au texte, Brossard, Linguatech, 241 p.] Meta, 48(4),

629-631. https://doi.org/10.7202/008743ar d'utilisation que vous pouvez consulter en ligne.

https://apropos.erudit.org/fr/usagers/politique-dutilisation/ 
Pour apprécier la valeur de ce livre et ses qualités pédagogiques, on ne peut s'empêcher de le comparer à d'autres ouvrages. La concurrence est vive dans le domaine économique, couvert par d'excellents ouvrages de référence. Notons, parmi beaucoup d'autres, le Dictionnaire commercial de l'Académie des sciences commerciales, le Dictionnaire de la comptabilité et des sciences de la gestion de P. Ménard, le Lexique d'économie, de Silem et Albertini ou le Dictionnaire économique et financier de Bernard et Colli, et dans une optique très pédagogique, le Dictionnaire d'apprentissage du français des affaires, de Binon et al.

L'ouvrage de J. Percebois ressemble plus en fait à l'excellent livre de F. Garrec, Commerce international et finance - International Trade and Finance (Economica,1991), qui met en contexte bilingue des textes traitant des grandes affaires économiques du jour, mais qui a déjà perdu un peu de son actualité, ou à celui de F. Renversez et al., Économie, dictionnaire encyclopédique (Dalloz, 1998).

Parmi les points forts, nous soulignons la formule originale qui présente en bilingue des textes d'auteurs (là où l'on trouve souvent des articles de journaux); mais nous déplorons le choix des extraits souvent très rébarbatifs car hors contexte et trop courts pour que le lecteur saisisse le fil conducteur. Ce problème, auquel s'ajoute la présentation non linaire des paragraphes, sur deux colonnes (anglais et français) et entrecoupés de notes terminologiques, rend la lecture extrêmement laborieuse. Par contre, les extraits sur les politiques, accords et institutions (moins théoriques) conviennent mieux à la formule du texte en traduction et sont tout à fait intéressants tant sur le plan du contenu que pour les problèmes de langue qu'ils illustrent.

Sur la forme, nous signalerons que l'on aurait pu accorder plus de soin à la traduction (parfois discutable ou trop littérale) et à la correction typographique, puisque de nombreuses fautes de frappe et de coupures de mots anglais ont échappé à la vigilance des réviseurs.

Pour conclure, le livre de J. Percebois mérite de s'inscrire dans la panoplie des ouvrages d'initiation à l'anglais économique par le texte. Toutefois, sa qualité pédagogique aurait pu être bien meilleure si la présentation avait été plus soignée. Du fait de son approche intéressante et originale, l'auteur aurait pu se tailler une place à part parmi les autres ouvrages couvrant ce domaine du commerce international où les besoins des apprenants sont si grands.

JeAnne E. Dancette

ETI, Genève, Suisse

Rouleau, M. (2001): Initiation à la traduction générale. Du mot au texte, Brossard, Linguatech, $241 \mathrm{p}$.

La publication, en 1958, de la Stylistique comparée du français et de l'anglais de Vinay et Darbelnet, œuvre pionnière, montrait la voie à l'enseignement universitaire de la traduction. Depuis, beaucoup d'auteurs, traducteurs ou traductologues, ont suivi le mouvement. On ne compte plus les publications d'ouvrages, rédigés dans la plupart des grandes langues de communication, visant à former des traducteurs, à les initier à l'art et à la pratique d'une activité attestée depuis la plus haute Antiquité. Depuis une vingtaine d'années, l'évolution vers la spécialisation du traducteur aidant, on assiste plus fréquemment à la parution d'ouvrages spécialisés (en commerce, finances et économie, publicité, droit, médecine, technique, etc.), voire théoriques, qu'à celle de manuels de formation à la traduction dite "générale».

C'est pourtant par là, avant de songer à se spécialiser, que le traducteur fera son apprentissage, acquerra les bases de son futur métier et prendra les bonnes habitudes qui lui serviront sa carrière durant. On ne le répètera jamais assez: avant de savoir traduire, il faut savoir écrire, et même bien écrire, préalable obligé à tout projet d'apprentissage de la traduction. Maurice Rouleau, auteur du premier (?) manuel pratique d'initiation à la traduction publié 
à l'aube du nouveau siècle, ne le voit pas autrement, pour qui «on ne s'improvise pas traducteur». Aussi, avant de prétendre devenir un spécialiste - objectif louable en soi - et de brûler des étapes, faut-il d'abord acquérir une solide formation générale. C'est le but que vise l'auteur de l'Initiation à la traduction générale, ouvrage qui est celui d'un pédagogue aussi responsable (cf. les avertissements lancés dans l'Avant-propos) que lucide ("traduire n'est pas aussi simple qu'il y paraît», p. xvii), doublé d'un praticien averti dont l'ambition est de former «un professionnel digne de ce nom» (p. xviii). Cette idée de professionnalisme sous-tend d'ailleurs l'ensemble de l'ouvrage. Car, comme chacun sait, la formation du traducteur est exigeante. Activité plus complexe qu'on ne le croit communément, traduire demande de la patience, beaucoup de rigueur et des connaissances quasi encyclopédiques. Avis à l'apprenti traducteur qui n'en tiendrait pas compte, la déconvenue sera à la hauteur de ses prétentions ou de sa naïveté.

Deux principes cardinaux ont inspiré la démarche de l'auteur. Celui de la connaissance et de la compréhension des langues et de leurs mécanismes, tout d'abord, lorsque l'auteur apostrophe ainsi le lecteur: «Êtes-vous bon récepteur?» Le second principe - «Êtes-vous bon émetteur?» - rappelle sans équivoque que la traduction est avant tout affaire de communication et qu'une communication efficace passe obligatoirement par la maîtrise du verbe. Les fondements de ce manuel d'initiation à la traduction générale reposent sur ces deux grands piliers. Sans pour autant prétendre avoir apporté des réponses à toutes les interrogations que se pose l'apprenti traducteur, l'auteur a su ramasser en quelques principes essentiels le contenu d'une riche et rigoureuse expérience. Les positions sont nettes, les réponses claires et les exemples nombreux, précis et pertinents.

L'ouvrage est divisé en cinq parties, dont trois principales, précédées d'une introduction. Le tout est assorti, à chaque étape de l'apprentissage, d'exercices d'application pertinents et fort bien conçus, ainsi que de figures illustrant le propos. Une série de douze annexes, consacrées à des difficultés particulières de la rédaction (typographie, reformulations, équivalences...), parachève un manuel principalement destiné aux étudiants du premier cycle universitaire. Un index des mots-clés permet au lecteur de retrouver facilement les noms et notions essentiels présentés. Dès l'introduction, ce dernier est mis devant ses responsabilités quand l'auteur lui propose d'emblée de devenir un spécialiste de la communication. Car c'est bien de communication qu'il s'agit quand on parle de la traduction "pragmatique», celle qui s'apparente, langue de départ en moins toutefois, au travail d'un bon journaliste.

Après les grandes lignes, passons à une revue plus détaillée de l'ensemble. Dans la première partie, l'auteur pose les données du problème, soit ce que l'étudiant doit savoir faire pour pouvoir traduire: connaître les langues en cause, les comprendre (savoir lire) et les faire comprendre (savoir rédiger). Dans un langage simple et clair, l'auteur expose les bases de ces trois conditions. Il va de soi que la «connaissance» des langues, pour un traducteur, va un peu plus loin que celle de Monsieur ou Madame Tout-le-monde. Il faut en maîtriser les différents codes (orthographe, grammaire, ponctuation, etc.), mais ne pas les confondre, notamment entre l'anglais et le français. On sait que la ponctuation, par exemple, ne joue pas tout à fait le même rôle dans les deux langues. La virgule dans les textes juridiques, notamment les lois, n'était pas interprétée de la même manière en anglais et en français, jusqu'à récemment en tout cas.

Ce constat doit rendre le traducteur d'autant plus prudent. La compréhension de ce que lit une personne est d'un autre ordre, elle dépend de sa capacité à déchiffrer les signes linguistiques et à en interpréter le sens. Pour le traducteur, c'est le texte qui mobilise son attention, entre autres sa fonction, sa forme, le sujet et le niveau de difficulté. Mais une chose est de comprendre ce que lit une personne, une autre sera de le faire comprendre à d'autres personnes. Là est toute la difficulté pour le traducteur, qui doit adapter la rédaction de son texte en fonction des servitudes (p. 37), dont la nature et la structure du texte de départ, tout en conservant une certaine latitude, celle que lui laisse la manière de rédiger, 
d'exprimer le message, donc le "sens", en français. La question de la longueur des textes, des phrases et paragraphes reste néanmoins posée. C'est celle de la lisibilité et de ses principes cartésiens: clarté, logique, concision (p. 40), dont doit tenir compte le traducteur de langue française.

La deuxième partie - «ce que vous devrez produire» - est la plus brève des trois. Elle traite uniquement des critères d'une bonne traduction, dont on sait que le nombre n'est pas infini. Mais c'est l'occasion pour l'auteur d'en rappeler les principaux (p. 51). Le traducteur, qui est aussi (surtout!) un rédacteur, révèle par cette opération l'aboutissement d'une formation, d'une compétence, mais réalisées dans un texte où le message prend forme. La restructuration éventuelle du message, la logique d'exposition des idées et la clarté de l'expression choisie sont autant d'obligations liant le traducteur dans sa performance, quelle que soit la forme du texte à traduire, bien ou mal rédigé. Dans la traduction communicative, différente de la littéraire par exemple, le traducteur se doit de faciliter la compréhension du message.

La troisième partie, la plus étoffée des trois, est celle qui a retenu plus particulièrement l'attention de l'auteur. C'est aussi celle où transparaît sa contribution la plus originale à la formation générale du traducteur. Le lecteur y trouvera quelques-unes des idées les plus personnelles de l'auteur, qui y traite en détail et de façon convaincante des éléments importants du texte que sont le mot, la phrase et le paragraphe, sans oublier le texte lui-même (p. 161) et son "fil conducteur». On pourrait aller jusqu'à dire que ces cent et quelques pages constituent un traité dans le traité sur l'art et la manière de (mieux) rédiger, mais à partir d'un texte de départ composé dans une langue autre, l'anglais en l'occurrence. De nombreux exercices, exemples et modèles ponctuent cette partie, car - air bien connu - c'est par la pratique (de la rédaction) que l'on devient rédacteur. On retiendra que l'auteur accorde à la formulation-réexpression du message toute l'importance qui doit lui revenir, peut-être au détriment de principes moins cardinaux.

Les deux dernières parties, la quatrième particulièrement qui récapitule les grands principes étudiés et traite du contrôle de la qualité, constituent la conclusion de l'ouvrage. La cinquième est un recueil de divers textes généraux destinés à présenter au futur traducteur, en le mettant en situation, la variété des domaines à traiter et des difficultés à affronter. On y verra la sollicitude d'un pédagogue soucieux de l'intérêt de ses étudiants autant que les conseils d'un professionnel d'expérience.

Ainsi la boucle est-elle bouclée et le parcours balisé. Concis et direct dans la forme, sans jargon et non dénué d'humour, cet ouvrage abonde en principes et conseils qui ne devraient pas laisser indifférent l'apprenti traducteur. Le lecteur n'y trouvera toutefois pas le traité d'un théoricien élaborant une méthode ou un système complexe. Il y puisera, plutôt, matière à réflexion sur la lettre et l'esprit de la traduction, ses applications, mais aussi sur le savoir-faire attendu du futur praticien, ainsi que sur les manières, parfois fort subtiles, de dire ou de ne pas dire les choses.

Jean-Claude Gémar ETI, Genève, Suisse

Kristal, E. (2002) : Invisible Work: Borges and Translation, Nashville, Vanderbilt University Press, $213 \mathrm{p}$.

Miroirs et labyrinthes, ruines et bibliothèques sont pareillement les thèmes de l'œuvre de Borges et les métaphores de sa poétique. Univers circulaire aux confins indistincts dans lequel se brouillent les repères d'une chronologie linéaire, s'estompe la position fondatrice de l'auteur, se noie le concept d'originalité. La critique littéraire a abondamment étudié ces 